\title{
Comentario al artículo de Ramón Ragués ${ }^{1}$
}

\author{
Luís Greco*
}

El estimulante artículo de Ragués propugna el reconocimiento de la nueva figura jurídica de la ignorancia deliberada, cuyo tratamiento debe ser idéntico a aquel conferido al dolo. Mis reflexiones tendrán por objeto principalmente la defensa de esta figura; pues un relevamiento exhaustivo de la bibliografía sobre el tema superaría los límites de un trabajo que se propone ser un mero comentario de otro artícu1o. Pretendo demostrar que, en buena parte de los casos en que fue reconocida por la jurisprudencia española, la figura es prescindible, porque en realidad, se debería hablar de dolo eventual (abajo 1). Los casos que tiene en mente Ragués, sin embargo, son aquellos en que el contenido de la figura excede el del dolo ("ignorancia deliberada en sentido estricto"); aquí, no obstante, ella me parece inaceptable (abajo 2). Por último, como consideración adicional, dotada de menor importancia científica pero de gran importancia práctica, tengo serias dudas respecto de la adecuación de la figura con el derecho positivo argentino o español (abajo 3).

* Privatdozenty Dr. Jur., Universidad de Ludwig Maximilian, Munich, Alemania.

1 Traducción de María Valentina Risso 


\section{El carácter en parte superfluo de la ignorancia deliberada}

En la mayoría de los casos en que la idea de la ignorancia deliberada ha sido reconocida, existe, en rigor, dolo. El primer caso español citado por Ragués (ítem IV.A) en que alguien que, acusado de encubrimiento por llevar una considerable cantidad de dinero en efectivo a un paraíso fiscal, alegó no saber que el dinero era proveniente del tráfico de drogas, existe dolo: el autor del encubrimiento no tiene que saber que la cosa obtenida es producto de este o de aquel delito, tan solo, que ella es producto de un delito ${ }^{2}$. Piénsese, también, en el caso en que alguien que acaba de hacerse un examen para detectar si es o no portador de una enfermedad contagiosa decide no abrir el sobre con el resultado del examen y sin conocer con seguridad el peligro que representa, termina contagiando a un tercero.

En esos casos, no hay mucho que discutir. En ellos, como precisa Ragués (ítem IV.A), existe indiscutiblemente dolo, al menos bajo la forma de dolo eventual ${ }^{3}$.

A menudo, el propio comportamiento que inmediatamente 4 causa el resultado ya irá acompañado de la conciencia de que algo puede terminar mal, de que posiblemente existe algún riesgo para un bien penalmente protegido. Esa conciencia será, en la mayoría de los casos, suficiente para justificar un dolo eventual. Aunque tenga por objeto solamente la posibilidad de un riesgo, posibilidad de un riesgo y existencia de un riesgo son nociones que, desde una perspectiva ex ante, se confunden. Y es esa la perspectiva decisiva al momento de evaluar el dolo, ya que el dolo constituye disvalor (subjetivo) de la acción. Desde una

2 Cf. por todos Altenhain, Nomos Kommentar zum Strafgesetzbuch, 4 ${ }^{\mathrm{a}}$. ed. Beck, Munique, 2013, § $259 \mathrm{~nm} .60$.

3 Suponiendo aquí que se acepta la distinción clásica entre las tres formas de dolo- en contra, con argumentos convincentes, Puppe, Nomos Kommentar zum Strafgesetzbuch, $4^{\text {a }}$. ed. Beck, Munique, 2013, $\S 15 \mathrm{~nm}$. 105 e ss.

4 Se entiende por "inmediatamente" aquel comportamiento que hace posible la realización del tipo sin que sea necesaria la práctica de otra conducta por el autor -algo así como el componente objetivo del concepto de tentativa acabada (sobre ese concepto, por todos, Roxin, Strafrecht Allgemeimer Teil, vol. 2, Beck, München, 2004, § 30 nm. 155). 
perspectiva ex ante solo hay posibilidades; lo real, en rigor, interesa solo en el momento de imputar el disvalor de resultado. Quien dispara contra una persona que tal vez parezca muerta, pero que en realidad está durmiendo, actúa con dolo de matar tanto si tuviera conocimiento de que la persona está viva, como si conociera -simplemente- esa posibilidad. Del mismo modo, el conocimiento de la posibilidad de contagiar a otra persona ya configura, pues, el elemento cognitivo del dolo de lesiones corporales. En estos casos, no existe ignorancia propiamente dicha.

En otros casos, el autor, en el momento en que practica ese comportamiento inmediatamente causante del resultado, no pensó siquiera en el riesgo o en la posibilidad del riesgo. En cierto modo, hay aquí ignorancia. En la mayoría de los casos, sin embargo, será posible identificar un comportamiento previo, en que ese conocimiento mínimo estaba presente y en que se tomó la decisión a favor de la ignorancia. Cuando esto ocurre, por regla será ese comportamiento anterior, y no el comportamiento inmediatamente causante de la realización del tipo, el comportamiento transgresor de la norma. Aquí, no hay más que una autoría mediata en que el autor hace uso de su yo futuro como instrumento que actúa con error excluyente del dolo. De la misma forma que el autor puede dar un revólver a un inocente X, garantizar que está descargado, y convencerlo a dar un susto a $\mathrm{Y}$, quien muere en virtud del disparo, el autor puede, al menos en teoría, hacer de sí mismo lo que hizo de X. Digo en teoría, porque es casi inimaginable que ese autor no se acuerde de su actuar previo. Si, en un ejercicio de imaginación jurídica, construyésemos un caso en que incluso ese recuerdo no existiera, no habría, en general, obstáculo para que se declare la decisión previa como la verdadera acción que realiza el tipo. Habrá aquí algo como una actio libera in causa, aunque poco típica, ya que el comportamiento previo no excluye, como en los casos prototipo de actio libera in causa, la culpabilidad del actuar posterior y sí su carácter doloso. De cualquier forma, en sustancia existe una actio libera in causa / una autoría mediata que, en el fondo, tampoco difiere sustancialmente de los casos en que el autor comete un delito a distancia. Quien coloca un reloj bomba en algún lugar puede estar 
dormitando al momento de la explosión; el conocimiento que fundamenta el dolo tiene que estar presente en el momento de la práctica de la acción que infringe la norma, esto es, en el momento en que la bomba es instalada.

Es decir: la mayor parte de los presuntos casos de ignorancia deliberada son casos en los que o bien no existe tal ignorancia, y si la acción que inmediatamente realiza el tipo es practicada con conciencia de la posibilidad de un riesgo, lo que es suficiente para el dolo eventual; o bien en que la posterior ignorancia es resultado de una situación anterior de conocimiento, de modo que el comportamiento previo justificará la responsabilidad por dolo, según los principios de la autoría mediata /de la actio libera in causa / del delito a distancia. Ya sea por vía directa o por vía indirecta, existe aquí dolo eventual y no ignorancia.

\section{La ignorancia deliberada en sentido estricto}

Los casos discutidos precedentemente, sin embargo, no son los que tiene en mente Ragués. El autor distingue la situación por él denominada de "ignorancia deliberada en sentido estricto" (ítem IV), que comprende los casos en que parece imposible justificar, incluso por vía indirecta, una imputación a título de dolo. Entre los ejemplos mencionados se encuentran el del director de una empresa que emite la orden a los empleados para que repelan las comunicaciones oficiales; o el de un director que difunde una dirección de e-mail para fines de comunicación de irregularidades en el funcionamiento de la empresa, e-mail este que, desde un principio, estaba dispuesto a no abrir nunca. También en el leading case, United States v. Jewell, parece difícil afirmar el dolo ${ }^{5}$ : quien recibe dinero para cruzar, con un automóvil, la frontera de México hacia los Estados Unidos, consciente de que el

$5 \mathrm{Al}$ menos desde nuestra perspectiva, que reconoce el dolo eventual. Dejo abierta la discusión si el término "knowing", usado por los tipos penales en cuestión, exige una forma calificada de dolo, como el dolo directo de segundo grado. 
automóvil tiene un compartimento secreto y de que en él hay alguna carga, puede hasta saber que algo anda mal; si se trata de 110 libras de marihuana como era el caso, o de armas, de pornografía infantil, o de órganos, él no tiene cómo saberlo. En esos casos, hay que reconocer la imposibilidad de fundamentar el dolo. Ragués considera, no obstante, que sería una respuesta inadecuada del ordenamiento castigar aquí únicamente por imprudencia o dejar al autor impune si el delito en cuestión ni siquiera reconoce la forma culposa. Sus argumentos, desarrollados en dos niveles, no me parecen del todo convincentes (abajo, a), b)). Además, la idea de un desconocimiento equivalente al dolo enfrenta un problema adicional, especialmente respecto al tratamiento de la tentativa (abajo c)).

a) El primer argumento lleva la discusión a un nivel que, realmente, no tiene cómo esquivarse: el de la razón de la punición por dolo. Ragués considera que el fundamento del tratamiento más severo conferido en el ordenamiento jurídico para los casos de dolo estaría en que el dolo presenta un contenido significativo distinto de la imprudencia (V. 1). El dolo es entendido como un desafío frontal a la vigencia de la norma. La imprudencia, por su parte, no solo no expresaría semejante mensaje, sino que en general expondría al autor a los peligros de una poena naturalis. Ragués afirma que ciertos casos de ignorancia deliberada, especialmente aquellos en que el autor se beneficia de la propia ignorancia -volveremos a este argumento enseguida- se situarían más próximos del dolo que de la imprudencia.

No cuestionaré el argumento en su dimensión empírica- sociológica $^{6}$ que , a primera vista, me parece plausible. La tesis contraria, de que la norma es desafiada con menor intensidad por el comportamiento practicado en estado de ignorancia es, no obstante, igualmente plausible, lo que ya insinúa que el problema no puede ser solucionado en el plano empírico. Pero incluso si se acepta la tesis empírica propuesta por Ragués, la perspectiva comunicativa por él defendida no me resulta convincente. Si lo que interesa es el conte-

6 Suponiendo que se trata de un modelo empírico. 
nido significativo del hecho, actos practicados a plena luz del día presentarían mayor contenido de injusto que los actos practicados en secreto. El llamado "crimen perfecto", que nadie descubre, ni siquiera existiría. Revelar un delito sería más grave que cometerlo. La razón por la cual el derecho prohíbe y castiga comportamientos no puede estar, pues, en lo que ellos comunican respecto de la vigencia de una norma y sí en el peligro que esos comportamientos representan para la subsistencia de un bien ${ }^{7}$.

Desde esta perspectiva, que no es la de Ragués, pero que me parece la más adecuada ${ }^{8}$, queda bastante clara la diferencia entre dolo e ignorancia deliberada. Quien actúa dolosamente, esto es, al menos con conocimiento de un riesgo, controla el riesgo inherente a su actuar, dispone de un cierto dominio que no está presente en quien no tiene ese conocimiento9: el conocimiento permite al autor adaptarse a nuevas circunstancias, reaccionar ante lo inesperado. Esto le da un control sobre la realización del tipo que otorga al ordenamiento dos razones para dirigir sus atenciones a tal comportamiento. En primer lugar, se trata de un comportamiento más peligroso, no para la vigencia de la norma y sí para la subsistencia de un bien; un legislador interesado en proteger bienes jurídico tendrá, pues, una razón de bien común, esto es consecuencialista para emplear sus escasos recursos preventivos -generales principalmente en el sentido de reprimir la práctica de ese comportamiento. En segundo lugar, cada quien es particularmente responsable de aquello que domina, porque esto tiene una íntima relación con su libertad, y existe como un principio fundamental, moral y jurídico, que correlaciona libertad y responsabilidad. De este modo, existe una razón no de bien común sino de respeto o deontológica en el sentido de una imputación más severa de quien actúa con conocimiento.

7 Esa crítica en Greco, Wider die jüngere Relativierung der Unterscheidung von Unrecht un Schuld, in: GA 2009, p. 636 e ss., 642 e s.

8 Cf. Greco, Lebendiges und Totes in Feuerbachs Straftheorie, Berlin, Duncker \& Humblot, 2009, p. 304 e ss.

9 Argumento formulado en Greco, Dolo sin voluntad, en: Silva Dias e otros (coords.), Liber Amicorum de José de Sousa e Brito, Coimbra, Almedina, 2009 , p. 885 e ss., p. 891 e s. 
En comparación, la perspectiva de Ragués parece insatisfactoria. Ella se ocupa únicamente del primer orden de consideraciones -de aquello que la sociedad obtiene por castigar más gravemente el dolo-, no importándole si la sociedad tiene o no derecho de buscar ese beneficio reprimiendo al autor. La afirmación de que la sociedad castiga para neutralizar el contenido de sentido expresado por el autor justifica la pena exclusivamente a partir de una perspectiva de la sociedad, mencionando un fundamento de interés social o de bien común. Y ella ni siquiera describe de modo adecuado el interés social que aquí se trata, el cual, como dije, es un beneficio referido a la subsistencia de bienes, y no a la vigencia de normas.

b) La tesis de la ignorancia deliberada como equivalente del dolo carece, pues, de un fundamento adecuado. Podríamos detenernos aquí. Sin embargo, Ragués arremete con un argumento dotado de plausibilidad intuitiva y que, por consiguiente, puede ser separado de la fundamentación expresivista más general (ítem V.2). Este argumento es la diferenciación, introducida por Luban, entre dos órdenes de razones para el desconocimiento: existen casos en que el autor se comportaría como un avestruz, en que el desconocimiento no equivaldría al dolo. Pero en los casos en que el autor se comporta como un zorro, sería el principal beneficiado de la ignorancia. No sería justo, pues, recompensarlo. Podríamos decir que, por el contrario, el autor estaría beneficiándose dos veces, primeramente por la mayor facilidad con que alcanzará su objetivo, en segundo lugar por escapar del castigo a título de dolo.

Reconózcase que el argumento tiene cierta plausibilidad intuitiva. Me parece, sin embargo, que esa intuición no pasa de ser un sentimiento impasible de verdadera justificación. Porque la distinción entre dolo e imprudencia no tiene nada que ver con la estructura más o menos altruista de la motivación del autor. Hay dolo altruista e imprudencia egoísta. El hecho de que alguien, a pedido de una víctima que no aguanta más las agonías de un cáncer que todavía puede acarrearle años, le administre una inyección letal, no excluirá el dolo de matar, de la misma forma que el hecho de que un médico desatento, en verdad, odiare al paciente no tendrá el poder de, por sí solo, justificar ese dolo. 
Motivaciones de un lado, dolo / imprudencia del otro, se encuentran en planos distintos. Estos últimos tienen que ver con el riesgo creado por el autor, es decir, guardan en última instancia una íntima relación con la idea de que el fundamento del injusto penal es la lesión a un bien jurídico; aquellos por su lado son a primera vista un cuerpo extraño en la teoría del injusto (y hasta de la culpabilidad), y solo recientemente se consiguió fundamentar de forma convincente su relevancia ${ }^{10}$. E1 hecho de que la perspectiva expresivista tienda a oscurecer esa distinción de planos es, en rigor, más una señal de cuanto esa perspectiva es insatisfactoria.

c) Por último, me gustaría presentar una objeción dogmática de carácter más general, que deberá ser superada por todo aquel que defienda la tesis de que sea igualada al dolo una situación en que no existe siquiera conocimiento. Los casos de imprudencia solo se castigan si se realizan todos los elementos del tipo; la presencia del dolo, por su parte, legitima la punición, aún careciendo de uno o más de dichos elementos, en este caso habrá tentativa. Si la ignorancia deliberada equivale al dolo, eso significa que, en los casos en que el tipo no se realice por completo el autor no quedará exento de pena y sí deberá ser castigado por la tentativa. Esto, sin embargo, genera una serie de dificultades que, a mi modo de ver, demuestran que la figura es, en el mejor de los casos, una imprudencia un tanto grave, pero nunca un dolo o algo a él equivalente.

Explicitemos estas dificultades con base en los tres casos encima mencionados de ignorancia deliberada en sentido estricto. Imagínese que, al final, nunca se dirigiera una comunicación oficial a los empleados que recibieron la orden de jamás tomar conocimiento de ella; o que nunca se escribiese un e-mail a la cuenta que fue instituida para nunca ser abierta. Si realmente se toma en serio que la ignorancia deliberada equivale al dolo, el emisor de la orden o quien creó la cuenta de e-mail tendría, aún así, que ser castigado por la tentativa. Ya eso parece un contra sentido. Piénsese también en el caso americano, United

${ }^{10}$ Me refiero a Peralta, Motivos reprochables, Marcial Pons, Madrid etc., 2012, p. 121 e ss. 
States v. Jewell, ahora sin modificación alguna: en rigor, no habría posibilidad alguna de castigar por el delito de transportar conscientemente sustancias estupefacientes. Como el autor deliberadamente ignoraba lo que transportaba, si armas, órganos, pornografía infantil, etc., él en rigor podrá ser castigado por tentativa de tenencia o trasporte de armas, órganos, pornografía infantil, etc. ${ }^{11}$.

Obsérvese que existen todavía tres dificultades adicionales. La primera respecto a la fijación del inicio de la tentativa; al no haber conocimiento, no hay plan del autor, punto de referencia con base al cual el inicio de la tentativa es generalmente fijado en la actualidad.

A esa dificultad súmase una incluso mayor, que es la de determinar el tipo cuya realización se está tentando. Y cuando no ocurre nada ¿tentativa de qué? ¿De homicidio, de lesiones, de daño, de estafa, de evasión fiscal, de contaminación de aguas? Si de alguna de ellas ¿de cuál? La única respuesta posible, una vez más, es: de todos.

Con eso llegamos a una tercera dificultad, de orden práctica o procesal. Los problemas apuntados no solo saltan a la vista en los casos americanos por razones procesales, una vez que el fiscal americano solo acusa por lo que él entiende necesario y el tribunal resuelve, en principio, solamente por la calificación escogida por el fis$\mathrm{cal}^{12}$. En un sistema procesal que reconoce el principio de legalidad procesal y la libertad del juez de recalificar jurídicamente los hechos objeto de la acusación, ellas se tornan insuperables no solo en teoría sino también en la práctica. Si aceptásemos la ignorancia deliberada, tendríamos que acusar por la tentativa de todos los tipos que podrían haberse realizado, e incluso los que la acusación no haya mencionado y condenar por todos ellos.

En fin, la idea de la ignorancia deliberada parece tener sentido cuando se piensa el delito desde una perspectiva ex post, a partir de que el resultado acontece. Si tenemos ya el transporte de marihuana,

${ }^{11}$ Suponiendo, obviamente, la existencia de tipos penales correspondientes y de la punibilidad de la tentativa.

${ }^{12}$ Sin embargo el problema subsiste, porque el fiscal tendría, en verdad, discrecionalidad para acusar por todas esas tentativas o para usarlas como moneda de cambio para constreñir al imputado a una declaración de culpabilidad. 
de armas, de órganos o de pornografía infantil, o la muerte de A, la lesión de $\mathrm{B}$, la destrucción de la propiedad de $\mathrm{C}$, la falta de pago de un tributo o el desecho de toxinas en un río, es fácil reconducir ese resultado a la decisión del autor de conocer al menos de la posibilidad de que ese resultado suceda. Pero la norma de comportamiento se formula no desde una perspectiva ex post sino ex ante; desde una perspectiva ex ante, en muchos casos difícilmente se podrá identificar de qué delito se trata, a no ser que se tenga en cuenta el conocimiento del autor.

Aquí se percibe bien por qué la ignorancia deliberada en sentido estricto solo pueda ser considerada imprudencia: si alguien viola su deber objetivo de cuidado/crea un riesgo jurídicamente desaprobado, por ejemplo, realizando un adelantamiento peligroso y nada ocurre, el derecho no lo castiga por la tentativa, también ${ }^{13}$ por la imposibilidad de distinguir aquí entre tentativa culposa de homicidio, lesiones o daño ${ }^{14}$.

\section{Barreras de lege data}

Por último, Ragués afirma que no existen obstáculos en el derecho español ni en el argentino para el reconocimiento de una ignorancia deliberada equiparable al dolo (V.2). Pienso que aquí Ragués no valoró en profundidad el texto de la ley, que tanto en España (art. 14.1 CP) como en Argentina (art. 34.1 CP) contienen una regla expresa sobre el error de tipo, que determina que el desconocimiento de elementos del tipo excluye el dolo. Me parece que esa regla, existente también en el derecho alemán (§ 16 I StGB) o en el brasilero (art. 20 caput CP), excluye de antemano la figura de la ignorancia deliberada en sentido estricto; reconocerla significa hacer una analogía contra reo, en violación al principio nullum crimen. Es curioso que Ragués ni siquiera mencione esos dispositivos y que no proponga una reinterpretación

${ }^{13}$ Los fundamentos más profundos de la no punibilidad de la tentativa culposa quedarán para otra oportunidad.

${ }^{14}$ Suponiendo que el ordenamiento castigue el daño culposo, lo que en general no sucede. 
"constructiva" 15 . De todos modos, vislumbro pocas posibilidades de releer dispositivos que dispongan que el error sobre el elemento del tipo excluya el dolo en el sentido de que errores sobre elementos del tipo justifiquen el dolo. La tesis de la ignorancia deliberada puede, como máximo, ser sustentada de lege ferenda, como propuesta de reforma legislativa.

${ }^{15}$ Como hace, por ejemplo, Pérez Barberá, El dolo eventual, Buenos Aires, Hammurabi, 2011, p. 50 e ss. -sin éxito, en mi opinión. 